Aksaray University
Journal of Science and Engineering
e-ISSN: 2587-1277
http://dergipark.gov.tr/asujse
$\underline{\text { http://asujse.aksaray.edu.tr }}$

Research Article

\title{
Gaussian Modified Pell Sequence and Gaussian Modified Pell Polynomial Sequence
}

\author{
Tulay Yagmur ${ }^{1, *}$, Nusret Karaaslan ${ }^{2}$ \\ 1,2 Department of Mathematics, Aksaray University, Aksaray, Turkey \\ ${ }^{1}$ Program of Occupational Health and Safety, Aksaray University, Aksaray, Turkey
}

-Received Date: 3 Jan 2018

-Revised Date: 06 Apr 2018

-Accepted Date: 10 Apr 2018

-Published Online: 25 June 2018

\begin{abstract}
In this paper, we first define the Gaussian modified Pell sequence, for $n \geq 2$, by the relation $G q_{n}$ $=2 G q_{n-1}+G q_{n-2}$ with initial conditions $G q_{0}=1-i$ and $G q_{1}=1+i$. Then we give the definition of the Gaussian modified Pell polynomial sequence, for $n \geq 2$, by the relation $G q_{n}(x)$ $=2 x G q_{n-1}(x)+G q_{n-2}(x)$ with initial conditions $G q_{0}(x)=1-x i$ and $G q_{1}(x)=x+i$. We give Binet's formulas, generating functions and summation formulas of these sequences. We also obtain some well-known identities such as Catalan's identities, Cassini's identities and d'Ocagne's identities involving the Gaussian modified Pell sequence and Gaussian modified Pell polynomial sequence.
\end{abstract}

\section{Keywords}

Modified Pell sequence, Modified Pell polynomial sequence, Gaussian modified Pell sequence, Gaussian modified Pell polynomial sequence.

* Corresponding Author: Tulay Yagmur, tulayyagmurr@gmail.com; tulayyagmur@aksaray.edu.tr 


\section{INTRODUCTION}

The complex Fibonacci numbers have been introduced by Horadam [1] in 1963. Then Berzsenyi [2] and Jordan [3] studied on Gaussian Fibonacci and Lucas numbers. The Gaussian Fibonacci numbers $\left\{G F_{n}\right\}_{n=0}^{\infty}$ are defined recursively by the relation $G F_{n}=G F_{n-1}+G F_{n-2}$ with initial conditions $G F_{0}=i$ and $G F_{1}=1$. Similarly, the Gaussian Lucas numbers $\left\{G L_{n}\right\}_{n=0}^{\infty}$ are defined as $G L_{n}=G L_{n-1}+G L_{n-2}$ where $G L_{0}=2-i$ and $G L_{1}=1+2 i$. Moreover, many authors studied on these numbers and their properties. For a little part of these studies, one can see, for example [4-6]. Halıcı and Öz [7] introduced the Gaussian Pell and Pell-Lucas numbers respectively by

$$
\begin{gathered}
G P_{0}=i, G P_{1}=1 ; G P_{n}=2 G P_{n-1}+G P_{n-2}, \\
G Q_{0}=2-2 i, G Q_{1}=2+2 i ; G Q_{n}=2 G Q_{n-1}+G Q_{n-2} .
\end{gathered}
$$

Moreover, Pell and Pell-Lucas polynomials are defined as

$$
\begin{gathered}
P_{0}(x)=0, P_{1}(x)=1 ; \quad P_{n}(x)=2 x P_{n-1}(x)+P_{n-2}(x) \\
Q_{0}(x)=2, Q_{1}(x)=2 x ; \quad Q_{n}(x)=2 x Q_{n-1}(x)+Q_{n-2}(x)
\end{gathered}
$$

respectively. Furthermore, Horadam and Mahon [8] gave some properties of these polynomials. Additionally, the modified Pell polynomials are defined recursively by the relation $q_{n}(x)=$ $2 x q_{n-1}(x)+q_{n-2}(x)$ where $q_{0}(x)=1$ and $q_{1}(x)=x$. The generating function of the modified Pell polynomials is

$$
M(t, x)=\frac{1-x t}{1-2 x t-t^{2}}
$$

Also, the Binet's formula of these polynomials is

$$
q_{n}(x)=x \frac{\alpha^{n}(x)+\beta^{n}(x)}{\alpha(x)+\beta(x)}
$$

where $\alpha=x+\sqrt{x^{2}+1}$ and $\beta=x-\sqrt{x^{2}+1}$ are the roots of the equations $r^{2}-2 x r-1=0$.

Then Halıcı and Öz [9] defined Gaussian Pell polynomial sequence as follow:

$$
G P_{0}(x)=i, G P_{1}(x)=1 ; \quad G P_{n}(x)=2 x G P_{n-1}(x)+G P_{n-2}(x) .
$$

The main objective of this paper is to define and study Gaussian modified Pell sequence and Gaussian modified Pell polynomial sequence.

\section{GAUSSIAN MODIFIED PELL SEQUENCE}

In this section, we first give the definition of the Gaussian modified Pell sequence, and then we obtain Binet's formula and generating function for this sequence. Moreover, we give some results related with the Gaussian modified Pell sequence.

Definition 2.1 The Gaussian modified Pell numbers $\left\{G q_{n}\right\}_{n=0}^{\infty}$ are defined, for $n \geq 2$, recursively by 


$$
G q_{n}=2 G q_{n-1}+G q_{n-2}
$$

with initial conditions $G q_{0}=1-i$ and $G q_{1}=1+i$.

Also, it is clear that

$$
G q_{n}=q_{n}+i q_{n-1}
$$

where $q_{n}$ is the $n$-th modified Pell numbers.

Now, we give the generating function for the Gaussian modified Pell sequence by the following theorem.

Theorem 2.2 The generating function of the Gaussian modified Pell sequence is

$$
G(x)=\frac{(1-x)+i(-1+3 x)}{1-2 x-x^{2}} .
$$

Proof. Let us write

$$
\begin{gathered}
G(x)=\sum_{n=0}^{\infty} G q_{n} x^{n}=G q_{0}+G q_{1} x+G q_{2} x^{2}+\ldots+G q_{n} x^{n}+\ldots, \\
2 x G(x)=2 G q_{0} x+2 G q_{1} x^{2}+2 G q_{2} x^{3}+\ldots+2 G q_{n-1} x^{n}+\ldots,
\end{gathered}
$$

and

$$
x^{2} G(x)=G q_{0} x^{2}+G q_{1} x^{3}+G q_{2} x^{4}+\ldots+G q_{n-2} x^{n}+\ldots
$$

Thus, we have

$$
G(x)\left(1-2 x-x^{2}\right)=G q_{0}+\left(G q_{1}-2 G q_{0}\right) x .
$$

Hence, we obtain

$$
G(x)=\frac{(1-x)+i(-1+3 x)}{1-2 x-x^{2}}
$$

The Binet's formula for the Gaussian modified Pell sequence is given by the following theorem.

Theorem 2.3 The $n$-th term of the Gaussian modified Pell sequence is by

$$
G q_{n}=\frac{\alpha^{n}+\beta^{n}}{\alpha+\beta}-i\left(\frac{\beta \alpha^{n}+\alpha \beta^{n}}{\alpha+\beta}\right)
$$

where $\alpha$ and $\beta$ are the roots of the equation $r^{2}-2 r-1=0$.

Proof. We know that the general solution for the recurrence relation is given by

$$
G q_{n}=c \alpha^{n}+d \beta^{n}
$$


for some coefficients $c$ and $d$.

The initial conditions imply that $G q_{0}=c+d$ and $G q_{1}=c \alpha+d \beta$.

Solving the system, we obtain

$$
c=\frac{1-\beta i}{2} \text { and } d=\frac{1-\alpha i}{2} .
$$

Thus, we get

$$
\mathrm{G} q_{\mathrm{n}}=\frac{\alpha^{\mathrm{n}}+\beta^{\mathrm{n}}}{\alpha+\beta}-i\left(\frac{\beta \alpha^{\mathrm{n}}+\alpha \beta^{\mathrm{n}}}{\alpha+\beta}\right)
$$

We now investigate some identities and properties of the Gaussian modified Pell sequence.

Theorem 2.4 Let $n$ and $r$ be two positive integers. Then Catalan's identity for the Gaussian modified Pell sequence is

$$
G q_{n+r} G q_{n-r}-G{q_{n}}^{2}=2(-1)^{n+1}(1-i)\left[1+\frac{(-1)^{r-1}\left(\alpha^{r}+\beta^{r}\right)^{2}}{4}\right] .
$$

Proof. By using the Binet's formula of the Gaussian modified Pell sequence, we get

$$
\begin{aligned}
G q_{n+r} G q_{n-r}-G q_{n}{ }^{2}= & {\left[\frac{\alpha^{n+r}+\beta^{n+r}}{\alpha+\beta}-i\left(\frac{\beta \alpha^{n+r}+\alpha \beta^{n+r}}{\alpha+\beta}\right)\right]\left[\frac{\alpha^{n-r}+\beta^{n-r}}{\alpha+\beta}-i\left(\frac{\beta \alpha^{n-r}+\alpha \beta^{n-r}}{\alpha+\beta}\right)\right] } \\
& -\left[\frac{\alpha^{n}+\beta^{n}}{\alpha+\beta}-i\left(\frac{\beta \alpha^{n}+\alpha \beta^{n}}{\alpha+\beta}\right)\right]^{2} \\
= & {\left[\frac{-4(-1)^{n}+2(-1)^{n-r}\left(\alpha^{2 r}+\beta^{2 r}\right)}{(\alpha+\beta)^{2}}\right]+i\left[\frac{4(-1)^{n}-(-1)^{n-r}\left(\alpha^{2 r+1}+\beta^{2 r+1}+\beta \alpha^{2 r}+\alpha \beta^{2 r}\right)}{(\alpha+\beta)^{2}}\right] } \\
= & {\left[\frac{-4(-1)^{n}+2(-1)^{n-r}\left(\alpha^{2 r}+\beta^{2 r}\right)}{(\alpha+\beta)^{2}}\right]+i\left[\frac{4(-1)^{n}-2(-1)^{n-r}\left(\alpha^{2 r}+\beta^{2 r}\right)}{(\alpha+\beta)^{2}}\right] . }
\end{aligned}
$$

Since $\alpha+\beta=2$, we obtain

$$
\begin{aligned}
G q_{n+r} G q_{n-r}-G q_{n}^{2} & =(-1)^{n+1}(1-i)+(1-i)(-1)^{n-r}\left[\frac{\left(\alpha^{r}+\beta^{r}\right)^{2}}{2}-(-1)^{r}\right] \\
& =2(1-i)(-1)^{n+1}\left[1-(-1)^{-r} \frac{\left(\alpha^{r}+\beta^{r}\right)^{2}}{4}\right] \\
& =2(-1)^{n+1}(1-i)\left[1+\frac{(-1)^{r-1}\left(\alpha^{r}+\beta^{r}\right)^{2}}{4}\right] .
\end{aligned}
$$

By setting $r=1$ in Theorem 2.4 , we obtain the following corollary which gives Cassini's identity of the Gaussian modified Pell sequence.

Corollary 2.5 For positive integer $n$, we have

$$
G q_{n+1} G q_{n-1}-G q_{n}^{2}=4(-1)^{n+1}(1-i)
$$


The following theorem gives d'Ocagne's identity involving the Gaussian modified Pell sequence.

Theorem 2.6 For positive integers $m$ and $n$, we have

$$
G q_{m} G q_{n+1}-G q_{n} G q_{m+1}=4(-1)^{n+1}(1-i) P_{m-n}
$$

where $P_{n}$ is the $n$-th Pell number.

Proof. By using the Binet's formula, we have

$$
\begin{aligned}
G q_{m} G q_{n+1}-G q_{n} G q_{m+1}= & {\left[\frac{\alpha^{m}+\beta^{m}}{\alpha+\beta}-i\left(\frac{\beta \alpha^{m}+\alpha \beta^{m}}{\alpha+\beta}\right)\right]\left[\frac{\alpha^{n+1}+\beta^{n+1}}{\alpha+\beta}-i\left(\frac{\beta \alpha^{n+1}+\alpha \beta^{n+1}}{\alpha+\beta}\right)\right] } \\
& -\left[\frac{\alpha^{n}+\beta^{n}}{\alpha+\beta}-i\left(\frac{\beta \alpha^{n}+\alpha \beta^{n}}{\alpha+\beta}\right)\right]\left[\frac{\alpha^{m+1}+\beta^{m+1}}{\alpha+\beta}-i\left(\frac{\beta \alpha^{m+1}+\alpha \beta^{m+1}}{\alpha+\beta}\right)\right] \\
= & {\left[\frac{2(\alpha-\beta)\left(\alpha^{n} \beta^{m}-\alpha^{m} \beta^{n}\right)}{(\alpha+\beta)^{2}}\right]+i\left[\frac{\left(\alpha^{2}-\beta^{2}\right)\left(\alpha^{m} \beta^{n}-\alpha^{n} \beta^{m}\right)}{(\alpha+\beta)^{2}}\right] } \\
= & \frac{\left(\alpha^{n} \beta^{m}-\alpha^{m} \beta^{n}\right)\left[2(\alpha-\beta)-i\left(\alpha^{2}-\beta^{2}\right)\right]}{(\alpha+\beta)^{2}} .
\end{aligned}
$$

Since $\alpha+\beta=2$ and $\alpha-\beta=2 \sqrt{2}$, we obtain

$$
\begin{aligned}
G q_{m} G q_{n+1}-G q_{n} G q_{m+1}= & \sqrt{2}(1-i)\left(\alpha^{n} \beta^{m}-\alpha^{m} \beta^{n}\right) \\
= & \sqrt{2}(1-i)(-1)^{n+1}\left(\alpha^{m-n}-\beta^{m-n}\right) \\
= & 4(-1)^{n+1}(1-i) P_{m-n}
\end{aligned}
$$

Theorem 2.7 The sum of the Gaussian modified Pell numbers is

$$
\sum_{k=1}^{n} G q_{k}=\frac{1}{2}\left(G q_{n+1}+G q_{n}\right)-1 .
$$

Proof. From the recursive relation related with the Gaussian modified Pell sequence, we can write

$$
G q_{n-1}=\frac{1}{2} G q_{n}-\frac{1}{2} G q_{n-2}
$$

Then we have

$$
\begin{aligned}
& G q_{1}=\frac{1}{2} G q_{2}-\frac{1}{2} G q_{0} \\
& G q_{2}=\frac{1}{2} G q_{3}-\frac{1}{2} G q_{1}
\end{aligned}
$$




$$
G q_{n}=\frac{1}{2} G q_{n+1}-\frac{1}{2} G q_{n-1} .
$$

Hence, we obtain

$$
\begin{aligned}
\sum_{k=1}^{n} G q_{k} & =\frac{1}{2}\left(G q_{n+1}+G q_{n}\right)-\frac{1}{2}\left(G q_{0}+G q_{1}\right) \\
& =\frac{1}{2}\left(G q_{n+1}+G q_{n}\right)-1
\end{aligned}
$$

which completes the proof.

The following corollary immediately follows from Theorem 2.7.

Corollary 2.8 For $n \geq 1$, we have

$$
\begin{aligned}
& \text { i) } \quad \sum_{k=1}^{n} G q_{2 k}=\frac{1}{2}\left(G q_{2 n+1}-1-i\right) \text {, } \\
& \text { ii) } \quad \sum_{k=1}^{n} G q_{2 k-1}=\frac{1}{2}\left(G q_{2 n}-1+i\right) .
\end{aligned}
$$

\section{GAUSSIAN MODIFIED PELL POLYNOMIAL SEQUENCE}

In this section, we first define the Gaussian modified Pell polynomials and then we give Binet's formula and generating function of this type polynomials. We also obtain some identities and properties of these polynomials.

Definition 3.1 The Gaussian modified Pell polynomials $\left\{G q_{n}(x)\right\}_{n=0}^{\infty}$ are defined, for $n \geq 2$, by the recurrence relation

$$
G q_{n}(x)=2 x G q_{n-1}(x)+G q_{n-2}(x)
$$

with initial conditions $G q_{0}(x)=1-x i$ and $G q_{1}(x)=x+i$.

It is obvious that if we take $x=1$, we obtain the Gaussian modified Pell sequence. Also, it is easy to see that

$$
G q_{n}(x)=q_{n}(x)+i q_{n-1}(x)
$$

where $q_{n}(x)$ is the $n$-th modified Pell polynomial.

Now, we aim to give generating function and Binet's formula for the Gaussian modified Pell polynomials. For this purpose, we shall prove the following theorems:

Theorem 3.2 The generating function of the Gaussian modified Pell polynomials is

$$
P(t, x)=\frac{(1-x t)+i\left(-x+t+2 x^{2} t\right)}{1-2 x t-t^{2}} .
$$


Proof. The generating function can be written as $P(t, x)=\sum_{n=0}^{\infty} G q_{n}(x) t^{n}$. Then we have,

$$
\begin{gathered}
P(t, x)=G q_{0}(x)+G q_{1}(x) t+G q_{2}(x) t^{2}+\ldots+G q_{n}(x) t^{n}+\ldots, \\
2 x t P(t, x)=2 x G q_{0}(x) t+2 x G q_{1}(x) t^{2}+2 x G q_{2}(x) t^{3}+\ldots+2 x G q_{n-1}(x) t^{n}+\ldots,
\end{gathered}
$$

and

$$
t^{2} P(t, x)=G q_{0}(x) t^{2}+G q_{1}(x) t^{3}+G q_{2}(x) t^{4}+\ldots+G q_{n-2}(x) t^{n}+\ldots
$$

So, we get

$$
P(t, x)\left(1-2 x t-t^{2}\right)=G q_{0}(x)+\left[G q_{1}(x)-2 x G q_{0}(x)\right] t
$$

Thus, we obtain

$$
P(t, x)=\frac{(1-x t)+i\left(-x+t+2 x^{2} t\right)}{1-2 x t-t^{2}}
$$

Theorem 3.3 The n-th term of the Gaussian modified Pell polynomials is

$$
G q_{n}(x)=x\left[\frac{\alpha^{n}(x)+\beta^{n}(x)}{\alpha(x)+\beta(x)}-i \frac{\beta(x) \alpha^{n}(x)+\alpha(x) \beta^{n}(x)}{\alpha(x)+\beta(x)}\right]
$$

where $\alpha$ and $\beta$ are the roots of the equations $r^{2}-2 x r-1=0$.

Proof. It is well-known that the general solution for the recurrence relation is given by

$$
G q_{n}(x)=c \alpha^{n}(x)+d \beta^{n}(x)
$$

for some coefficients $c$ and $d$.

By considering the initial conditions, we get $G q_{0}(x)=c+d$ and $G q_{1}(x)=c \alpha+d \beta$.

Solving the system above, we obtain

$$
c=\frac{1}{2}-i \frac{\beta}{2} \text { and } d=\frac{1}{2}-i \frac{\alpha}{2} .
$$

Thus, we have

$$
G q_{n}(x)=x\left[\frac{\alpha^{n}(x)+\beta^{n}(x)}{\alpha(x)+\beta(x)}-i \frac{\beta(x) \alpha^{n}(x)+\alpha(x) \beta^{n}(x)}{\alpha(x)+\beta(x)}\right] .
$$

We now investigate some identities and properties of the Gaussian modified Pell polynomials.

Theorem 3.4 Let $n$ and $r$ be two positive integers. Then Catalan's identity for the Gaussian modified Pell polynomials is 


$$
G q_{n+r}(x) G q_{n-r}(x)-G q_{n}{ }^{2}(x)=2(-1)^{n+1}(1-x i)\left[1+\frac{(-1)^{r-1}\left(\alpha^{r}(x)+\beta^{r}(x)\right)^{2}}{4}\right] .
$$

Proof. By using the Binet's formula of the Gaussian modified Pell polynomial sequence, we get

$$
\begin{aligned}
G q_{n+r}(x) G q_{n-r}(x)-G q_{n}^{2}(x)= & {\left[x \frac{\alpha^{n+r}(x)+\beta^{n+r}(x)}{\alpha(x)+\beta(x)}-i x\left(\frac{\beta(x) \alpha^{n+r}(x)+\alpha(x) \beta^{n+r}(x)}{\alpha(x)+\beta(x)}\right)\right] } \\
& \times\left[x \frac{\alpha^{n-r}(x)+\beta^{n-r}(x)}{\alpha(x)+\beta(x)}-i x\left(\frac{\beta(x) \alpha^{n-r}(x)+\alpha(x) \beta^{n-r}(x)}{\alpha(x)+\beta(x)}\right)\right] \\
& -\left[x \frac{\alpha^{n}(x)+\beta^{n}(x)}{\alpha(x)+\beta(x)}-i x\left(\frac{\beta(x) \alpha^{n}(x)+\alpha(x) \beta^{n}(x)}{\alpha(x)+\beta(x)}\right)\right]^{2} \\
= & x^{2}\left[\frac{-4(-1)^{n}(1-x i)+2(-1)^{n-r}\left(\alpha^{2 r}(x)+\beta^{2 r}(x)\right)(1-x i)}{(\alpha(x)+\beta(x))^{2}}\right] .
\end{aligned}
$$

Since $\alpha+\beta=2 x$, we obtain

$$
\begin{aligned}
G q_{n+r}(x) G q_{n-r}(x)-G q_{n}^{2}(x) & =(-1)^{n+1}(1-x i)+\frac{(1-x i)(-1)^{n-r}\left[2 x^{2}\left(\alpha^{r}(x)+\beta^{r}(x)\right)^{2}-4 x^{2}(-1)^{r}\right]}{(\alpha(x)+\beta(x))^{2}} \\
& =-2(1-x i)(-1)^{n}\left[1-\frac{(-1)^{-r} x^{2}\left(\alpha^{r}(x)+\beta^{r}(x)\right)^{2}}{4 x^{2}}\right] \\
& =2(-1)^{n+1}(1-x i)\left[1+\frac{(-1)^{r-1}\left(\alpha^{r}(x)+\beta^{r}(x)\right)^{2}}{4}\right]
\end{aligned}
$$

By taking $r=1$ in Theorem 3.4, Cassini's identity involving the Gaussian modified Pell polynomials, which is given in the following corollary, is obtained.

Corollary 3.5 For positive integer $n$, we have

$$
G q_{n+1}(x) G q_{n-1}(x)-G q_{n}^{2}(x)=2\left(x^{2}+1\right)(-1)^{n+1}(1-x i) .
$$

d'Ocagne's identity involving the Gaussian modified Pell polynomials is given in the following theorem.

Theorem 3.6 For positive integers $m$ and $n$, we get

$$
G q_{m}(x) G q_{n+1}(x)-G q_{n}(x) G q_{m+1}(x)=2\left(x^{2}+1\right)(-1)^{n+1}(1-x i) P_{m-n}(x),
$$

where $P_{n}(x)$ is the n-th Pell polynomial.

Proof. By using the Binet's formula, we have

$G q_{m}(x) G q_{n+1}(x)-G q_{n}(x) G q_{m+1}(x)$ 


$$
\begin{aligned}
& =x^{2}\left[\frac{\alpha^{m}(x)+\beta^{m}(x)}{\alpha(x)+\beta(x)}-i\left(\frac{\beta(x) \alpha^{m}(x)+\alpha(x) \beta^{m}(x)}{\alpha(x)+\beta(x)}\right)\right]\left[\frac{\alpha^{n+1}(x)+\beta^{n+1}(x)}{\alpha(x)+\beta(x)}-i\left(\frac{\beta(x) \alpha^{n+1}(x)+\alpha(x) \beta^{n+1}(x)}{\alpha(x)+\beta(x)}\right)\right] \\
& -x^{2}\left[\frac{\alpha^{n}(x)+\beta^{n}(x)}{\alpha(x)+\beta(x)}-i\left(\frac{\beta(x) \alpha^{n}(x)+\alpha(x) \beta^{n}(x)}{\alpha(x)+\beta(x)}\right)\right]\left[\frac{\alpha^{m+1}(x)+\beta^{m+1}(x)}{\alpha(x)+\beta(x)}-i\left(\frac{\beta(x) \alpha^{m+1}(x)+\alpha(x) \beta^{m+1}(x)}{\alpha(x)+\beta(x)}\right)\right] \\
& =x^{2} \frac{\left[\alpha^{n}(x) \beta^{m}(x)-\alpha^{m}(x) \beta^{n}(x)\right]\left[2(\alpha(x)-\beta(x))-i\left(\alpha^{2}(x)-\beta^{2}(x)\right)\right]}{(\alpha(x)+\beta(x))^{2}} \\
& =\sqrt{x^{2}+1}\left[\alpha^{n}(x) \beta^{m}(x)-\alpha^{m}(x) \beta^{n}(x)\right](1-x i) \\
& =2\left(x^{2}+1\right)(-1)^{n+1}(1-x i) P_{m-n}(x) .
\end{aligned}
$$

Theorem 3.7 The sum of the Gaussian modified Pell polynomials is

$$
\sum_{k=1}^{n} G q_{k}(x)=\frac{1}{2 x}\left[G q_{n+1}(x)+G q_{n}(x)-x-1+i(x-1)\right] .
$$

Proof. From the recursive relation related with the Gaussian modified Pell sequence, we can write

$$
G q_{n-1}(x)=\frac{1}{2 x} G q_{n}(x)-\frac{1}{2 x} G q_{n-2}(x)
$$

Then we have

$$
\begin{aligned}
G q_{1}(x) & =\frac{1}{2 x} G q_{2}(x)-\frac{1}{2 x} G q_{0}(x) \\
G q_{2}(x) & =\frac{1}{2 x} G q_{3}(x)-\frac{1}{2 x} G q_{1}(x) \\
G q_{3}(x) & =\frac{1}{2 x} G q_{4}(x)-\frac{1}{2 x} G q_{2}(x) \\
\vdots & \\
G q_{n}(x) & =\frac{1}{2 x} G q_{n+1}(x)-\frac{1}{2 x} G q_{n-1}(x)
\end{aligned}
$$

Hence, we obtain

$$
\begin{aligned}
\sum_{k=1}^{n} G q_{k} & =\frac{1}{2 x}\left(G q_{n+1}(x)+G q_{n}(x)\right)-\frac{1}{2 x}\left(G q_{0}(x)+G q_{1}(x)\right) \\
& =\frac{1}{2 x}\left[G q_{n+1}(x)+G q_{n}(x)-x-1+i(x-1)\right]
\end{aligned}
$$

which completes the proof.

From Theorem 3.7, we can give the following corollary.

Corollary 3.8 For $n \geq 1$, we have

$$
\text { i) } \quad \sum_{k=1}^{n} G q_{2 k}(x)=\frac{1}{2 x}\left(G q_{2 n+1}(x)-x-i\right) \text {, }
$$


ii) $\quad \sum_{k=1}^{n} G q_{2 k-1}(x)=\frac{1}{2 x}\left(G q_{2 n}(x)-1+x i\right)$.

\section{CONCLUSION}

In this study, we introduce the concept of the Gaussian modified Pell sequence and Gaussian modified Pell polynomial sequence. We also give some results, such as Binet's formulas, generating functions, summation formulas for these sequences. Moreover, we obtain some well-known identities, such as Catalan's, Cassini's, d'Ocagne's identities involving these sequences.

\section{REFERENCES}

[1] A.F. Horadam, Complex Fibonacci Numbers and Fibonacci Quaternions. American Math. Monthly 70 (1963) 289-291.

[2] G. Berzsenyi, Gaussian Fibonacci Numbers. Fibonacci Quarterly 15(3) (1977) 233-236.

[3] J.H. Jordan, Gaussian Fibonacci and Lucas Numbers. Fibonacci Quarterly 3 (1965) 315318.

[4] J.J. Good, Complex Fibonacci and Lucas Numbers, Continued Fractions, and the Square Root of the Golden Ratio. Fibonacci Quaterly 31(1) (1981) 7-20.

[5] C.J. Harman, Complex Fibonacci Numbers. Fibonacci Quaterly 19(1) (1981) 82-86.

[6] S. Pethe, A.F. Horadam, Generalized Gaussian Fibonacci Numbers. Bull. Austral. Math. Soc. 33(1) (1986) 37-48.

[7] S. Halıc1, S. Öz, On Some Gaussian Pell and Pell-Lucas Numbers. Ordu Univ. Science and Technology Journal 6(1) (2016) 8-18.

[8] A. F. Horadam, J. M. Mahon, Pell and Pell-Lucas polynomials. Fibonacci Quarterly 23(1) (1985) 7-20.

[9] S. Halıc1, S. Öz, On Gaussian Pell Polynomials and Their Some Properties. Palestine Journal of Mathematics 7(1) (2018) 251-256. 